\title{
Aperçu sur la littérature des Juifs Espagnols.
}

Les Juifs d'Espagne, particulièrement ceux des royaumes de Castille et d'Aragon, conquis par les rois chrétiens, remplacèrent bientôt la langue arabe qu'ils parlaient, par la langue du pays, c'est-àdire la langue espagnole ou castillane. Déjà au milieu du treizième siècle elle était si générale parmi les Juifs, que non sculement les savants, les médecins et les rabbins s'en servaient. On sait que R. Moseh de Nachman ou Nachmanide soutint dans la langue du pays, la défense du judaïsme contre Pablo Christiani, Juif converti, en présence du roi Jacques d'Aragon et des grands seigneurs du royaume. Aussi les Juifs qui ne comprenaient pas l'hébreu en faisaient-ils usage même dans lc culte divin: dans quelques communautés israélites de Castille et d'Aragon le rôle d'Esther fut lu publiquement en langue espagnole à la fête de Purim. 1

Sous le règne d'Alphonse $\mathrm{X}$, surnommé le Savant, qui fit bien des efforts afin de purifier la langue castillane des éléments arabes et romans, quelques Juifs s'essayèrent comme écrivains dans la langue vulgaire. Les premiers essais littéraires des Juifs espagnols sont des traductions de l'arabe en castillan, qu'ils composèrent par ordre du roi: Fuda de Moseh Colen, un Juif nommé Abraham, Yshac Ibn Sid et Semuel ha.Levi traduirent pour lui des ouvrages astronomiques et en composèrent quelques uns eux-mêmes.

Sous le successeur d'Alphonse nous trouvons deux Juifs: $\mathscr{F} a 00 b$ de Las Leyes et Facob Zaddik de Ucles, qui composèrent, ou mieux compilèrent, comme Fehuda de Astruc de Barcelone, des oeuvres philosophiques en castillan, et Abraham Ibn Hayim écrivit un traité espagnol sur la préparation de l'or et des teintures appliquées à la peinture dans les livres. Aussi les traités sur le jeu des échecs de Abraham Ibn Esra et Moseh Acan de Zaragua furent traduits en espagnol.

17a. לקיטות הרמב"ז מגילד (Salonique 1780). (Communiqué par Mr. L. Blau, professeur à Budapest.) 
Quelque temps après des Juifs espagnols rivalisèrent avec les troubadours. Le premier Juif qui composa des vers pleins de grace en langue castillane fut $\mathrm{R}$. Santob de Carrion, connu sous le nom de Rabbi Don Santo, cité par les écrivains espagnols comme un grand troubadour; il a mis en vers des «Proverbios morales», tirés de l'Ecriture sainte et du Talmud.

Après la terrible persécution en 1391 bien des Juifs acceptèrent le baptême, entre lesquels un assez grand nombre cultivant la littérature espagnole. Le poète Juan Alphonso de Baena est l'auteur d'un recueil intitulé "Cancionero", qui contient des poésies de divers auteurs, entre autres de Pcro Fcrrus, Fuan de España, Fuan de Valladolid, Antonio lie Montoro cel Roperos; ils étaient, comme Baena et Rodrigo Cota, le premier' dramaturge espagnol, des Juifs baptisés.

Mais aussi des fidèles adhérents du judaïsme écrivircnt en même temps en langue espagnole. D. Chasdai Crescas et Foseph Albo, deux philosophez bien connus, composèrent des traités polémiques contre le christianisme. D. Mlosih Zar zal, médecin du roi Henri III, fit des vers à l'occasion de la naissance de Juan II, roi d'Espagne. D. Neeir Alguadt'z, médecin et rabbin, composa un traité médical; Semuel Esperial et Selomol Marik composèrent des traités de chirurgie. Des parties des ouvrages de Galen, de Lanfranc, de Théo. doric de Cervie e. a. furent traduits, ainsi qu'un opuscule ssur la peste, de Jean de Tornamira, et elas Especias de litargirio», qui fut traduit ou transcrit en espagnol. Tous les ouvrages précités furent écrits en caractères hébraïques et probablement en Espagne.

La reine Isabelle bannit en 1492 les Juifs de l'Espagne; D. Ma. nuel, qui employait des astronomes juifs, tels que foseph Vecinho, son médecin, et Abrahan Zacuto, son astrologue et chroniste, auteur d'ouvrages astrologiques, les chassa bientôt après du Portugal.

Les fugitifs se refugièrent en Italie, en France, dans les diverses provinces de la Turquie; les Pays-Bas et l'Angleterre, Hambourg et Vienne accordèrent plus tard asile à ceux qui, persécutés par l'inquisition, quittèrent les pays intolérants. Partout ils emportèrent avec eux la langue maternelle. "Llevaron de acá», dit Gonzalo de Illescas, "nuestra lengua y todavia la guardan y usan della de buena gana; $\mathrm{y}$ es cierto que en las ciudades de Salonique, Constantinopla, Alesandria y el Cairo y en otras ciudades de contratacion y en Venecia no compran ni venden ni negocian en otra lengua sino en español. $\mathrm{Y}$ yo conoci en Venecia hartos Judios de Salonique que hablaban castellano, con ser bien mozos, tambien ó mejor que yo.»

Ainsi que la langue, les Juifs espagnols ont conservé les cou. tumes espagnoles; les romances, les chansons, les mélodies et les proverbes nationaux, même le menu des Juifs d'origine espagnole existent encore aujourd'hui en Espagne. 
Les Juifs espagnols en Bulgaric ont par exemple le chant populaire suivant: 1

\author{
Mi padre era di Francia \\ mi madre d'Aragon: \\ por ser yo regalado \\ di chica mi caso; \\ mi caso con un Franco, \\ hijo d'un grand' señor; \\ non lo quiero, non lo quiero yo, etc. \\ De onde es esta zamaro, \\ que aqui veyo yo? \\ Yo lo mando mi padre \\ de la feria d'Aragon, etc. \\ Arvolera! Arvolers! \\ la rama era d'uro, \\ y la raiz de marfil, etc.
}

Des vaudcvilles espagnols avec les mélodies, oubliés depuis longtemps en Espagne, se sont conservés en grand nombre chez les Juifs bannis. On les trouve comme titres de poèmes hébreux, composés par de pieux poc̀tes en Turquie et en Italie. Nous en donnons les suivants: ${ }^{2}$

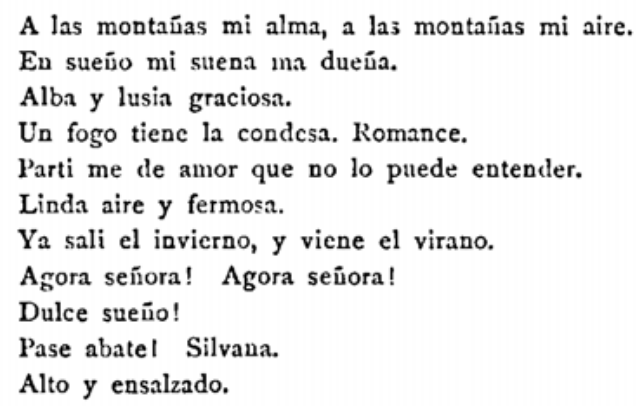

Quant aux proverbes, encore en usage parmi les Juifs espagnols, qui se sont transmis de génération en génération, nous en offrons, en appendice, pour la première fois un recueil. ${ }^{3}$

Les Juifs espagnols ont conservé les jeux d'enfants espagnols; par exemple cel Castillo', un jeu de noix, où on place une noix sur

1 Communiqué par Mr. S. F. Rosanes à Roustchouk; v. Istoria pe scurt a evreilor Bulgari: Annuar pentru Israeliti, an. XI (Bucuresti 1888), p. 14, I5.

2 V. Israel Nagara, Semiroth Israel (Venise 1599).

3 Le recueil de ces proverbes je le dois à mon cher ami Mr. S. H. Davitcho, consul de Serbie à Budapest, qui a été secondé dans son travail par ses frères à Belgrade, et à Mr. Facob $S$. Cohen à Roustchouk, qui a bien voulu me fournir quelyues matériaux. 
trois autres; ainsi qu'un jeu où l'on se range l'un après l'autre en se couvrant les yeux et où l'on chante:

\author{
Venid, venid Cavallero \\ assuvid al trionastero \\ escuje cuala quereis \\ a la bella non mi tomeis. 1
}

Les termes usuels pour les utensiles de cuisine, tel que rallo (râpe), trebedes (trèpieds), pala (pelle), parrillas (gril), padilla (poëlon), escoba (balai) et beaucoup d'autres, qui sont nommés souvent dans les codes rituels des Juifs, les vêtements et le menu - tout est espagnol.

Le menu. le plus favori du Juif espagnol, qui est devenu proverbial, est le 'pastel, un pâté au fromage ou à la viande; aagristada est une sauce d'oeufs aigris au citron; le ‘Pan d'España», appelé aussi "Pandelon», qui veut dire «Pan de Leon», se trouve régulièrement chez les Juifs en Serbie et en Bulgarie. ${ }^{1}$

Il est superflu de dire que les Juifs expulsés ne négligèrent pas la langue maternelle et qu'ils cultivèrent la littérature espagnole partout où ils s'établirent.

Nous trouvons en Italie, à Ferrare, les premiers fruits de leur diligence scientifique, et les premiers ouvrages qui y parurent étaient produits par la nécessité: un livre de prières quotidiennes et la traduction de la Bible, connue sous le nom de la Bible de Ferrarc; tous les deux parurent en 1553 aux dépens et dans l'imprimerie de Yom Tob Atias ou Abraham Usque et de Jeronimo de Vargas ou Duarte Pinel. Dans la même année y parut en portugais le livre en partie historique «Consolaçam as Tribulaçoês de Ysrael „, dont l'auteur, Scmuel Usque, parent de l'imprimeur ci-nommé, avait le but de con. soler ses frères malheureux et de les attacher à la foi paternelle, en leur racontant les persécutions qu'ils avaient souffertes. Salomon Usque, de la même famille, fit paraître en même temps les poésies de Pétrarque en traduction espagnole. Quinze ans plus tard deux ouvrages philosophiques parurent à Venise: l'un de Léon Alravanel et l'autre de Aron Afia, médecins et philosophes. Excepté les ouvrages philosophiques de Herrera et ceux de Vellosino et Orobio de Castro qui combattirent le spinozisme, ce sont les seules oeuvres des Juifs qui existent en langue espagnole ou portugaise.

Quant à la philosophie religieuse, Facob Abendana traduisit en r663 le «Cuzary, en espagnol, et David Nieto, l'auteur du ‘Cuzary second,, polémisa contre les Karaïtes et les adhérents de Descartes.

Les Juifs qui, persécutés par l'inquisition, suivirent par la suite des temps leurs coreligionnaires, ne comprenaient plus la langue

1 Communiqué par Mr. S. F. Rosanes à Roustchouk. 
hébraïque. Cette circonstance donna sujet à plusieurs rabbins et hommes doctes de traduire des ouvrages éthiques de l'hébreu en espagnol ou d'en composer des nouveaux. Nous ne devons pas passer sous silence que les règles sur l'abattage des bestiaux et que le "Sulhan Aruch,, ce conducteur sûr pour la vie religieuse, parurent transcrits en types espagnols pour la première fois à Venise en 1609 et plus tard souvent encore. Quelques années avant une traduction espagnole de la Mischna y avait paru et, un an après, celle du livre moral de Bahya Ibn Bakoda, intitulé :Obligacion de los corazones»; celle du eMenorat ha-Maor, ou 'Almenara de la luz', livre moral et ascétique très estimé, fut imprimée en 1656 à Livourne, et en 1654 y parut le traité sur la prière de David Mugnon. En 1627 Yshac Athias fit paraître à Venise son (Thesoro de preceptos,, Ahraham Farrar à Amsterdam la sdéclaration des 613 commandements de la loi écrite, et $S$. Gallego sous le titre «Sendroe de Vidas, la traduction d'un traité ascétique de $\mathrm{R}$. Jona de Gironde.

Depuis le commencement du dix-septième siècle la littérature juive espagnole prit un nouvel essor à Amsterdam. Cette ville franche qui ouvrait ses portes aux persécutés, devint bientôt l'asile d'un grand nombre de Marranes, d'hommes distingués, médecins, religieux, poc̀tes, savants et hommes d'Etat. Favorisée et protegée par de riches Juifs, les Pinto, les Belmonte, les Suasso, les Acosta, les D'Andrade, les Teixeira et beaucoup d'autres, la littérature trouva ses protecteurs dans toutes ses branches. Des Juifs espagnols établirent à Amsterdam des imprimeries, dont quelques unes, comme les officines de Menasseh ben Israel, Joseph Athias, David de Castro Tartas, Jacob de Cordova, Moseh Dias, Moseh Coutinho, acquirent une grande renommée et dans lesquelles des Juifs espagnols avaient été occupés comme imprimeurs et correcteurs. A Amsterdam il y avait même des libraires espagnols, qui étaient écrivains et éditeurs. Les imprimeurs d'Amsterdam fournissaient longtemps à presque tous les Juifs espagnols des traductions de la Bible, des prières, de la Mischna, et particulièrement du traité qui est chez eux en usage religieux; tous ces livres parurent très souvent en éditions nouvelles, améliorées et augmentées.

Dans les productions littéraires des Juifs espagnols, principalement à Amsterdam et à Hambourg, les oeuvres qui prennent le premier rang sont les écrits propres à fortifier les coreligionnaires dans la foi ou pour défendre le judaïsme et les dogmes juifs contre les attaques qu'ils soutinrent dans ce temps en dehors et au milieu de la propre communauté. Uriel da Costa, qui niait l'immortalité de l'âme, trouva un violent adversaire dans le médecin Semuel de Silva, traducteur du traité de la sTesuvah, de Maimonide (1613), qui l'attaqua en 1623 dans son sTratado da immortalidade del almas; le même sujet Menasseh ben Israel defendit en 1636. David Cohen 
de Lara traduisit en espagnol quelques traités de morale, et Seloinol de Oliveyra sous le titre •Ensenha à pecadores» un traité de l'ouvrage ascétique de Isaie Hurvitz. Yshac Fesurun à Hambourg est l'auteur d'un ouvrage sur la l'rovidence divine, qui fut publié après sa mort en I663, ct quelques ans après parurent le travail de Moseh Gideon Abudientc sur le Messie, et les ouvrages de morale de Abraham Israel Pereyra, Juif espagnol riche et bienfaisant.

Très grand est le nombre des écrits apologétiques et polémiques, composés par des Juifs qui s'étaient enfuis d'Espagne et de Portugal et s'étaient établis en Italie et dans les Pays-Bas, et dont la plus grande partie, c'est-à-dire les écrits de Montalto, de Morteira, de Orobio de Castro, de Pizarro, et de plusieurs écrivains anonymes, sont encore en manuscrit. Aux ouvrages imprimés appartiennent la -Nomologias de Imantel Aboab (1629), l'oeuvre de fuan Carrascon et d'Abraham Piregrino (1633), "las humildas suplicaciones» de Me. nassel ben Isracl (1655), «Fucnte Claras, clas Excelencias y Columnias de los Hebreos, de Yshac Cardoso (1679), et la «Repuestas de David Nicto (1723). A ce genre se range aussi le "Tratado da calumnia de B. Nahmias de Castro (1629), et la traduction de l'ouvrage polémique de Josèphe contre $\Lambda$ pion, faite par le capitaine Foscph Semalh Arias (1687).

Les travaux espagnols et portugais du ressort de la Halacha ou de la pratique religieuse sont fort peu nombrcux. Si nous exceptons l'ouvrage volumineux intitulé . Thesouro dos Dinim, de licnasseh ben Israel et le "Tratado de Herem» ou de "Halissa» de Rodrigo de Castro, ils se restraignent à quelques extraits du eSulhan Aruch. de Moseh de Toledo (1643), de David Pardo (1648) et à une com. pilation de Abraham Vaez (1692). D'autant plus grand est le nombre des sermons et oraisons. des oraisons funcibres et d'occasion; plus de soixante de ces sermons et oraisons parurent séparement et en recueil depuis la fin du dix-septième siècle jusqu'à notre temps. En Orient les rabbins prononcent leurs sermons encore à présent communément dans la langue judéo-espagnole qui est celle qu'ils comprennent.

Ajoutons les livres pour l'instruction de la jeunesse dans les principes de la foi dont quelques uns en forme de dialogue; citons ceux de Sonza Brito (1722), de Yiuda Leon Perez (1729), de Yshac de Paz, en hébreu et en espagnol (1764), de Facob Saruco et Yshac de Leon (i 766 ).

L'étude de la Sainte Ecriture ne fut pas négligée. Le Pentateuque fut traduit de nouveau par Menasseh ben Israel (1627), dont le .Conciliador», ouvrage en quatre parties, est très estimé, et par Foseph Franco Serrano qui en donna un commentaire concis (1695). Des paraphrases au Pentateuque furent fait par Yshac Aboab da Fonseca (1681) et Moseh Dias (1697). Yshac Acosta, Haham à Bayonne, com. 
posa les «Conjecturas Sagradas s sur les Prophètes. Les Psaumes furent traduits en prose par Facob Yeuda Leon Tcmplo avec un commentaire (1671), et en vers par David Abenatar Melo (1626) et par Daniel Israel Lopez Laguna (1720). Fuan Pinto Delgado traduisit en vers les lamentations de Jérémie, l'histoire dc Ruth et d'Esther (1627); et Abraham Lañado traduisit déjà en 1619 la paraphrase chaldaïque des Cantiques.

On trouvc aussi quelques grammairiens parmi les Juifs espagnols. En effet des grammaires hébraïques en langue portugaise ont été écrites par Moseh Gideon Abudiente (1633), Moseh Rephael de Aguilar (1660), Selomoh de Oliveyra (1681, 1682) et Selomoh Rephacl Feh. Leon Templo (1703). Facob Lumbroso composa, déjà en $\mathrm{I}_{588}$, un glossaire espagnol des mots difficiles de la Bible, et Léon Modena en 1675 un glossaire hébreu-espagnol; ces deux ouvrages ont eu plusieurs éditions. Facob Mantino est l'auteur d'un vocabulaire arabeespagnol, Selomoh de Olivej'ra d'un vocabulaire hébreu-portugais (1683), et Facob Rodrigues Moreira d'un vocabulaire hébreu-espagnol-anglais (1773). Abraham de Fonseca écrivit en 1663 une sOrthographia Castellana.s Abraham Meldola composa en ${ }_{17} 8_{5}$ à Hambourg une nouvelle grammaire portugaise.

L'intérêt pour l'histoire universelle en général n'était pas très grand chez les Juifs du temps antérieur, et était de peu d'importance chez les Juifs espagnols. Nous possédons un ouvrage important, composć par Mosch Almosnino et traduit par facob Cansino sous le titre ¿Extremos y grandezas de Constantinoplas (1638). Les descriptions sommaires de quelques villes par Daniel Levi de Barrios sont de peu de valeur historique. Des révélations remarquables sur le procédé de l'inquisition et ses victimes ont été données, longtemps avant Llorente, par le Haham David Nieto à Londres (1722). Quant à l'histoire juive, la chronique de Ibn Verga fut traduite en espagnol par Méir de Leon en 1640. L'ouvrage déjà mentionné de Semuel Usque est une source historique de valeur. Les ouvrages polémiques de Imanuel Aboab, de Menasseh ben Israel ct de Yshac Cardoso contiennent beaucoup de remarques historiques. David Franco Mendes et le fils d'Uri Levi ont laissé des mémoires sur l'établissement des Juifs à Amsterdam, et Daniel Levi de Barrios a publié des relations sur les institutions bienfaisantes et les différentes académies de la communauté sus-nommée. "L'affaire d'assassinat rituel de Yshac Jesurun, par Aron Cohen de Raguse est aussi traduite en espagnol. Pedro Teixeira nous donne des relations sur son voyage aux Indes Orientales (1610), et Moseh Pereyra de Paiva sur son séjour chez les Juifs en Cochinchine. Les relations sur les dix tribus de Israël par Antonio Montezinos furent citées par Menasseh ben Israel dans son ouvrage souvent traduit \&Esperança de Israel, (1650). Un 'conducteur' composa en espagnol Yshac de Lara. Comme auteurs des libres de Cosmographie et des cartes géographiques sont nommés 
David Nasi et Nicolas de Oliver y Fullana à Amsterdam, qui ont écrit des parties de la "Geographia Blaviana». Gabriel de Souza Brito est l'auteur d'un \&Epitome cosmografico, et d'un "Norte mercantils.

Quant aux sciences mathématiques nous avons à nommer un ouvrage non publié du "Comte palatin. Imanuel Rosales, et «Praxe da Arithmeticas de Facob Saruco (1766); celui de Facob Ribero de Paiva existe encore en ms. A l'astrologie appartient le cTratado das Cometas, de Imanuel Rosales, publié encore pendant son séjour à Lisbonne. Plusieurs Juifs espagnols s'occupèrent du calendrier et du calcul du temps, tel que Dicgo Barrassa (1630), Abraham Vecino, qui composa un calendrier astronomique de 1626 jusqu'à i666. Aron Franco Pinhero est l'auteur d'un \&Lunario perpetuos (1657). Selomoh de Oliveyra, qui écrivit sur les Tequphot (r687), composa un :Calendario de las Tablas Lunares.s David Nieto, Moseh de Foseph Belmonte, Yeuda Piza et beaucoup d'autres composèrent des calendriers.

Facob Yeuda Leon, appelé Tcmplo à cause du modèle qu'il avait construit du temple de Salomon, s'occupait des antiquités judaïques. Ses ouvrages, très estimćs de ses contemporains, concernant le Tabernacle, le temple de Salomon, les Chérubins, ne furent pas tous écrits par l'auteur en espagnol.

Les médecins juifs d'origine espagnole ou portugaise: Amato et Zacuto Lusitano, Ezcchiel et Rodrigo de Castro, les Fonseca, les Bueno, les Silva écrivirent, comme la plupart des médecins de ce temps, en langue latine. Yshac Cardoso publia encore pendant son séjour à Madrid quelques traités de médecine en espagnol, et $F a c o b$ de Castro Sarmento en composa et traduisit de l'anglais en portugais.

C'est surtout la poésie espagnole qui fut cultivée par les Juifs longtemps aprèz leur exil d'Espagne. Amsterdam, cette riche ville de commerce, l'Eldorado des Juifs pendant le dix-sept et le dixhuitième siècle, forma le centre des poètes juifs. ${ }^{1}$ Ifiguel ou Dantẹl Levi de Barrios, qui a conquis une place d'honneur parmi les auteurs dramatiques espagnols, l'auteur de "Flor de Apolos, de cCoro de las Musass, ce poc̀te pauvre et malheureux, qui devint l'historio. graphe de la communauté israélite portugaisc d'Amsterdam, nous a laissé une «Relacion de los Poetas españoles, à Amsterdam; en vérité une belle partie d'histoire de la littérature espagnole. ${ }^{2}$ Les premiers fondateurs de la dite communauté rendaient hommage à la poésie. Facob Israel Belinonte et Moseh Bclmonte, son fils, firent des vers contre l'inquisition. Francisco de Caceres écrivit les ‘Dialogos satiricos. (1616) et traduisit de l'italien, en 1612 , un ouvrage sobre la creacion

1 Voyez m. Sephardim. Romanische Poesien der Juden in Spanien. (Leipzig 1859):

2 La eRelacions avec une courte biographie du poète a été publiée par moi dans la Revue des études juives, t. 18, p. 276 et suiv. 
del mundo', qu'il dédia à facob Tirado, un des co-fondateurs de la communauté. Paul de Pina ou Reluuel fessurun, un jeune moine qui vint en 1604 à Amsterdam et qui conçut dix ans plus tard le premier règlement de sépulture pour la communauté, composa en portugais en 1624 le «Dialogo dos Montess, qui y fut représenté dans la synagogue de ‘Beth Jacob». Manuel Thomas, frère de Jonas Abravanel, poète et éditeur, est l'auteur de $O$ Phenix da Lusitania, et de cInsulanas, deux poèmes épiques, chacun de dix chants. Trois poc̀mes hćroïques sont publiés par des poètes juifs: „David, par Facob Usiel, qui parut en 1624 , la même année. où Imanuel Bocarra Frances y Rosales publia son “Anacephalaeosis»; "El Machabeos par Miguel de Silveyra (1637), et ‘El Samson Nazareno» par Antonio Enriquez Gomez (1657). Celui-ci qui se fit un nom par ses poésies épiques, lyriques et dramatiques, fut brûlé en effigie à Seville en I660; huit ans avant son ami Manuel Fernandez de Villa-Real, écrivain politique et militaire, fut garotté à Lisbonne. Villa-Real et autres martyrs de l'inquisition furent chantés par des poètes juifs, qui se trouvaient non seulement dans la vie civilc, mais aussi parmi les militaires, parmi les femmes, dont quelques unes se sont distinguées par leurs poésies, telles que Isabcl Correa, la traductrice espagnole du "Pastor Fido", Isabel Henriques, célébrée par son génie extra. ordinaire dans les académies de Madrid.

On trouvait aussi des académies poétiques à Amsterdam. Le . Comte Palatin. Manucl de Belmonte érigea en 1676 une académie, dont les juges étaient Yshac de Rocamora, avant sa rentrée au judaïsme prédicateur de Marie, impératrice d'Autriche, et Yshac Gomez de Sosa, poète latin. De Barrios, Moseh Dias, imprimeur et auteur, et Abraham Gomez Silveyra, poète et auteur de ‘Sermones s, en exerçaient les fonctions de smantenedoress ou défenseurs. Facob Antonio de Castillo, Abrahan Gomez de Arauxo et autres enobles géniess s'y distinguèrent par leurs énigmes et les explications qu'ils en donnèrent.

Une autre académie se forma à Amsterdam en 1685 sous le nom "Academia de los Floridos", qui a été décrite par Danicl Levi de Barrios. Lui et Simon Levi de Barrios, son fils, étaient mantenedores, avec le médecin Abraham Gutierrez, avec Duarte Lopes Rosa et Manuel de Leäo, deux poètes très doués et productifs. Foseph Penso Vega, homme de connaissances étendues, poète et orateur, auteur d'ouvrages moraux et philosophiques et de nouvelles, remplit les fonctions de secrétaire, et Moseh Orobio de Castro. médecin comme son illustre père, remplit celles de procureur fiscal. Les membres les plus importants de cette académie étaient les suivants: Yshac Orobio de Castro, D. Manuel de Belmonte, Geronimo Nunez de Acusti, Foseph Nunez Marchena, sdel Sabio luz, de la Academia adarga,, D. Francisco de Lis, "que á las Musas alcança, para que la 
flor lleva su alabanças, Mainuel Levi appelé Valle, rmonte de las Musass, Abraham Penso, frère de Joseph Penso Vega, Foseph Israel Alvarcz, cla historia exalta en su elegante escriptos, fosepll fesurun Lobo, consul du roi d'Espagne en Zélande, Facob de Chaves, celegante plumas, Semuel de Leon, c. a.

Le développement de la littérature parmi les Juifs en Hollande et en Angleterre cessa avec le temps où ils s'approprièrent la langue du pays, qu'ils finirent par regarder et aimer comme leur patrie. Toutefois nous $\mathrm{y}$ trouvons dans la première moitié du dixhuitième siècle quelques poètes, tel que fosé Henriquez de Almeyda, Bernard Nuñez Garcia, Felix et David Valle Saldaña, ou quelques écrivains comme Foseph Dacosta, l'auteur d'un .Tratado de Cortesias, et Yshac Fundam, libraire de livres espagnols, qui publia \&Entretenimientos en varios entremeses; mais nous trouvons aussi des Juifs espagnols qui écrivaient en hollandais, en français et en anglais. La langue espagnole, mais non la littérature espagnole s'est conservée parmi eux.

Pendant que les Juifs instruits faisaient usage de la langue espagnole pure et non corrompue en Italie, au sud de la France et en Hollande, un dialecte plus ou moins corrompu se formait chez les Juifs en Orient ct dans les pays contigus. Ce jargon est nommé ordinairement "Ladino (לאדינו), ou judéo-espagnol, ou "Ladino españols, elengua sephardis, ou elengua vulgar»; les Juifs dans les pays des Balcans le nomment. "Judesmos.1

Le Ladino diffèrc de l'espagnol par beaucoup de mots et de formes qui étaient en usage dans le castillan vers la fin du quinzième siècle, lorsque les Juifs furent bannis d'Espagne, et encore plus tard, p. e.: fincamiento, fijo, facer, fablar, daquen, vueso, espuegro (suegro), et autres. En Ladino on trouve souvent le changement de $\mathrm{m}$ avec $\mathrm{n}$, comme muestros, mos; l'intercalation $d u m$ et $n$, p. e.: amvisar, munchos, au lieu de avisar, muchos; le metathése du d avant $r, p$. e.: vedrad (verdad), vedre (verde), acodro (acordo), pedrer (perder). On trouve dans le Ladino beaucoup de termes et de phrases hébraïques ainsi que les mots adoptés de l'hébreu et espagnolisés; il y a des mots, fréquemment employés en Ladino, qui sont étrangers en espagnol, p. e.: 'meldar, lire, apprendre, "meldadors, lecteur, 'meldas, école, ou ‘darsan», du mot hébreu דרש, qui signifie prêcher, faire un sermon, e. a.

1 Mr. S. F. Rosanes à Roustchouk est d'opinion que Ladino signifie Latino. Lorsque șous l'empire romain la langue hébraïque cessa d'être une langue vivante, les Juifs, pour enseigner la Bible à leurs enfants, se servirent d'une langue qui leur était compréhensible, c'est-à-dire du latin; de là on prit l'habitude de dire au lieu de traduire de l'hébreu slatinar ou sladinars, ou cenladinars. Plus tard on dit pour traduire en esprignol emeldar en Ladinos. 
Le Ladino, en général imprimé en types rabbiniques et ëncore à présent écrit avec les signes hébraïques, nommés cécriture espagnoles, possède une littérature à lui qui prit le commencement bientôt après l'expulsion. Le premier écrit des Juifs' d'Espagne, qui parut déjà en $\mathrm{I} 5 \mathrm{IO}$ et qui provint immédiatement des besoins quotidiens, fut la traduction des règles sur l'abattage des bestiaux conforme à la loi; il suivit la traduction de la Bible, de toute la Sainte Ecriture et de ses livres secondaires, principalement de ceux dont on fait usage dans le culte public, des psaumes, des rôles ou Megillot, surtout du Cantique avec la paraphrase chaldaïque, qui furent plusieurs fois réimprimés et traduits de nouveau. Le livre de Sirach ne fut traduit en Ladino qu'en 1818 par Israel Bahor Haim de Belgrade, qui a beaucoup de mérite en littérature judéo-espagnole. Ainsi que la Bible, les prières quotidiennes et occasionelles, les prières des fêtes, le récit de Pesach, appelé la Hagadah, et autres furent traduits en Ladino; des prières faites aux événements extraordinaires comme celles qui ont été récitées à l'anniversaire du tremblement de terre de Livourne (1742) sont traduites. Les sentences des anciens rabbins, le travail poétique des six cent treize commandements de la loi, le plus ancien des poésies de la synagogue, ne furent pas seulement traduits plusieurs fois, mais aussi munis d'un commentaire très diffus (1777).

Pour faire connaître la pratique religieuse et ceremonielle, aux hommes qui ne savaient pas la langue hébraïque, et surtout pour les femmes, on fit des extraits du livre Sulhan Aruch dè Joseph Caro et les traduisit en judéo-espagnol (1 568, 1602); de cet ouvrage toute la partie, concernant le culte intérieur, fut traduite en $\mathrm{I} 744$ et dans les temps modernes.

Une éthique homilétique avec un traité sur les songes fut composée par Moseh Almosnino, rabbin et prédicateur renommé à Salonique, en 1564 ien romances, qui fut réimprimée en 1729; du même auteur parut l'ouvrage historique déjà mentionné, que facob Cansino a transcrit en 1638 en lettres espagnoles. Yshac de Paz.composa en I764 en hébreu et en judéo-espagnol un livre de réligion, contenant les treize articles de foi, la signification des jours de fête et de jeùne et la déclaration des dix commandements. On traduisit en Ladino des ouvrages moraux très répandus: les .Devoirs des Coeurs, de Bahya Ibn Bakoda, le «Menorat ha-Maor» de Yshac Aboab, le livre ascétique de Elie de Vitas, les ssentences de la viè de Elieser de Worms, et le livre estimé ‘Kaw Hajaschar, de H. Kaidenower, ainsi que quelques libres cabalistiques.

Les femmes de l'Orient n'aiment pas la lecture, et les beaux arts n'ont pas $\in u$ place dans la littérature judéo-espagnole jusqu'aux temps récents, pourtant ‘Esther , la tragédie de Racine, et des histoires diverses ont été traduites; d'autant. plus riche est elle en chants Coplas, Cantares, Roxas - pour la fête dc Purim, avec des. jeux poétiques et petites comédies aux divertissements de fête $(1700-1868)$. 
Quant aux productions poétiques, nous mentionnons l'histoire di Joseph, composée en 1732 par Abraham de Toledo, et quelques chansons nationales, entremêlées de mots hébreux et espagnols. Nous communiquons comme exemple la chanson suivante: 1

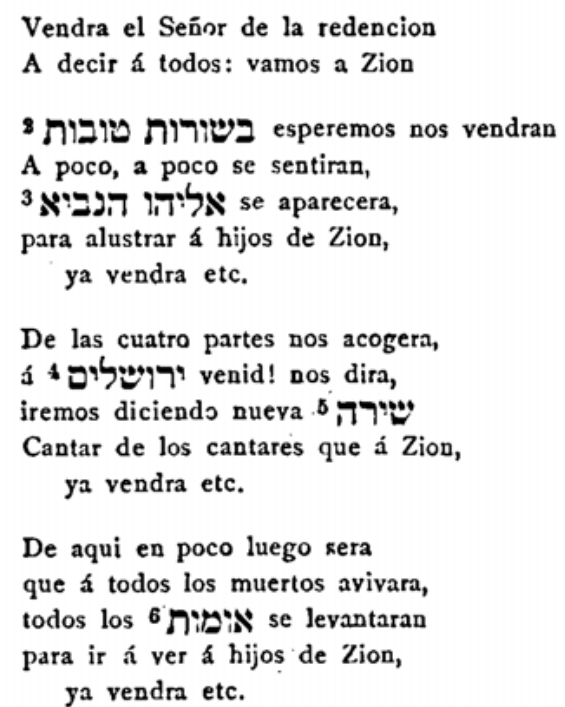

Ya vendra Moseh el pastor ${ }^{7}$ נמאט, que por su 8 ia descendo 90 y agora presto vendra el 10 pror. de rescatar á hijos de Zion, ya vendra etc.

Le livre destiné pour le peuple, l'ouvrage historique de Joseph ben Gorion, cy resto de historias verdaderas s, trouva déjà, avant le milieu du siècle passé, un traducteur en Ladino.

La littérature judéo-espagnole prit un nouvel essor, lorsque les Juifs en Orient commencèrent, il y a quelques dizaines d'années, de s'occuper de la culture et des sciences. Depuis ce temps les officines de Vienne, de Belgrade, principalement cclles de Constantinople, de Salonique et de Smyrne fournirent aux Juifs un assez grand nombre d'ouvrages judéo-espagnols. On ne se borna pas seulement à traduire la Bible et les prières, les livres et discours moraux, les extraits du "Sohar; ; on commença de composer des grammaires, un vocabulaire hébreu-ladino, une histoire universelle, une géographie, une astronomie, même un livre d'arithmétique, des biographies de

1 פישיר מאפן (Amsterdam 1793); v. Orient (1844) p. 683, comm. par A. Ink. (Jellinek). Des autres chansons se trouvent: Sephardim 142 et suiv.

2 Mensages buenos. - ${ }^{3}$ Elia el profeta. - 4 Jerusalem. - 5 Cancion. - 6 Pueblo. - 7 Fiel. -8 Merito. - 9. Maná. - 10 Tiempo. 
coreligionnaires célèbres. Pour défendre le judaïsme contre les attaques des adversaires et des missionaires, comme M'Caul, dont le cold Paths, fut réfuté, on traduisit aussi l'ouvrage ancien ‘Fortification de la foi, ( 1840 ), et pour répandre la connaissance des persécutions et des souffrances des ancêtres, on transcrivit le «Vara de Jehuda, en Ladino (1850. 1859). A l'usage de la jeunesse et du peuple on traduisit le traité sur les animaux de Kalonymos, l'histoire de cmille et une nuits, ou on en composa de nouveaux, ainsi l'histoire de Joseph, d'Esther etc.

$\mathrm{Ce}$ qui est très caractéristique pour la culture des Juifs en Orient, c'est qu'il a paru, il y a vingt-cinq ans à Smyrne, un opuscule soi-disant médical, ne contenant que des remèdes sympathiques. L'ouvragc le plus important de la littérature judéo-espagnole est celui qui parut sous le titre "Meam Loës, un commentaire encyclopédique sur le Pentateuque, le livre de Josué et le rôle d'Esther, composé par différents auteurs, ouvrage qui a eu plusieurs éditions.

Les journaux périodiques qui paraissent à Belgrade, à Constantinople, Salonique, Smyrne etc., les statuts et bulletins des communautés israélites, de leurs écoles, de leurs instituts de bienfaisance sont presque tous écrits en judéo espagnol, qui, chez les Juifs en Orient, est toujours la langue vulgaire. Aucune autre langue n'était pas en état de déplacer entièrement la langue du pays que les juifs aimaient à travers les siècles: la langue espagnole. 
